\title{
FEDSM2008-55266
}

\section{NUMERICAL STUDY OF TURBULENCE MODELS IN THE COMPUTATION OF BLOOD FLOW IN CANNULAS}

\author{
Felix A. Salazar \\ Universidad Simón Bolívar, \\ Grad Student, Mechanical \\ Engineering \\ e-mail: felix.salazar@usb.ve
}

\author{
Luis R. Rojas-Solórzano \\ Universidad Simón Bolívar \\ Dpto. de Conversión y Transporte de \\ Energía \\ e-mail: rrojas@usb.ve
}

\author{
James F. Antaki \\ Carnegie Mellon University \\ Dept. of Biomedical Engineering \\ e-mail: antaki@andrew.cmu.edu
}

\begin{abstract}
In recent years, CFD has become an increasingly used tool in the design of blood-based devices. However, the estimation of red blood cells damage (hemolysis) remains a very important challenge due to the complex rheology of blood and the turbulence present in most pumping devices. The objective of this study was to identify an appropriate turbulence model suitable for predicting hemolysis in Hemodialysis cannula. Several modern turbulence models were evaluated in comparison to Direct Numerical Simulation (DNS), which was used as the gold standard. The fluid dynamics in the cannula was modeled as a coaxial jet in which Reynolds' number approached 2800. Based on comparison of velocity and stress time-averaged profiles, the Shear Stress Transport (SST) model with Gamma-Theta transition was identified as an optimal compromise between accuracy and computational cost.
\end{abstract}

\section{NOMECLATURE}

CFD Computational Fluid Dynamics

DNS Direct Numerical Simulation

RANS Reynolds Averaged Navier-Stokes

SST Shear Stress Transport

$\triangle P f H b \quad$ Variation of plasma-free-hemoglobin [mg/L]

$\mathrm{Hb} \quad$ Total amount of hemoglobin [mg/L]

$\tau \quad$ Shear stress $[\mathrm{Pa}]$

$t \quad$ Time [s]

$\bar{U} \quad$ Velocity vector $[\mathrm{m} / \mathrm{s}]$

$D_{L} \quad$ Linear blood damage [dimensionless]

A Diameter of cannulas' tip [mm]

$B \quad$ Diameter of cannulas' bore [mm]

$x, y, z \quad$ Cartesian coordinate directions [mm]

\begin{tabular}{|c|c|}
\hline & Radius [mm] \\
\hline$U_{x}(r)$ & $\begin{array}{l}\text { Streamwise component }(x \text { direction) of the inlet } \\
\text { velocity profile }[\mathrm{m} / \mathrm{s}]\end{array}$ \\
\hline$U_{x}(x, r)$ & $\begin{array}{l}\text { Streamwise component ( } x \text { direction) of the velocity } \\
\text { vector, at an axial position } x \text { and radius } r[\mathrm{~m} / \mathrm{s}]\end{array}$ \\
\hline$U_{1}$ & $x$ velocity of the inner jet at the inlet $[\mathrm{m} / \mathrm{s}]$ \\
\hline$U_{2}$ & $x$ velocity of the outer jet at the inlet $[\mathrm{m} / \mathrm{s}]$ \\
\hline$U_{3}$ & $x$ velocity of the free-stream at the inlet $[\mathrm{m} / \mathrm{s}]$ \\
\hline$R_{1}$ & Radius of the inner jet [mm] \\
\hline$R_{2}$ & Radius of the outer jet [mm] \\
\hline $\operatorname{Re}_{R_{1}}$ & Inner jet diameter Reynolds' number. \\
\hline$R_{M}$ & Average radius of the jets $[\mathrm{mm}]$ \\
\hline$\theta_{01}$ & $\begin{array}{l}\text { Momentum thickness of the inner shear layer of } \\
\text { the jet array at the inlet [mm] }\end{array}$ \\
\hline$\theta_{02}$ & $\begin{array}{l}\text { Momentum thickness of the outer shear layer of } \\
\text { the jet array at the inlet [mm] }\end{array}$ \\
\hline 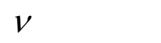 & Kinematic viscosity $\left[\mathrm{m}^{2} / \mathrm{s}\right]$ \\
\hline$v_{T}$ & Turbulent viscosity $\left[\mathrm{m}^{2} / \mathrm{s}\right]$ \\
\hline$k$ & Turbulent kinetic energy $\left[\mathrm{m}^{2} / \mathrm{s}^{2}\right]$ \\
\hline$\varepsilon$ & Turbulent dissipation $\left[\mathrm{m}^{2} / \mathrm{s}^{3}\right]$ \\
\hline$\omega$ & Turbulent frequency $\left[\mathrm{s}^{-1}\right]$ \\
\hline$r_{\max }(x)$ & $\begin{array}{l}\text { Radius where the streamwise velocity component } \\
\text { reaches its maximum [mm] }\end{array}$ \\
\hline$U_{x_{\max }}(x)$ & $\begin{array}{l}\text { Maximum of the streamwise velocity component } \\
{[\mathrm{m} / \mathrm{s}]}\end{array}$ \\
\hline$\delta(x)$ & Thickness of the jet [mm] \\
\hline
\end{tabular}




\section{INTRODUCTION}

The blood stream in arteries and veins is put under a shear stress, which depends on the flow parameters, and is transferred to the cellular constituents of the blood. These stresses will distort the shape of the cellular membrane, and under certain conditions, could provoke the rupture of the membrane. If this happens to a red blood cell, the hemoglobin will be released into plasma, phenomenon which is called hemolysis [1].

The study of this phenomenon is critical to the design of biomedical devices, such as blood pumps, dialysis machines, heart valves, catheters and cannulas, among others. The in-vitro evaluation of these devices is the most reliable way to perform the hematologic study [2]. However, it requires great number of repetitions, due to statistical variations, to assure the validity of the results. These experiments are quite expensive, increasing the design and development cost of the blood-based medical devices.

As a result, the hemolysis analysis by means of computational fluid dynamics (CFD) appears as an attractive alternative [3-5], because it could decrease the cost and time of design and development of medical devices. With CFD analyses, accurate calculation of hydrodynamic variables, such as velocity, pressure and stress fields could be obtained for the devices under study. According to typical cannula flow rates, the Reynolds number may overcome the turbulence threshold in certain regions. Nevertheless, previous CFD studies have been limited the study to laminar regimes. Due to the presence of flow transition from laminar to turbulent regime, and the turbulence itself within cannulas, it becomes mandatory the use of a turbulence model when calculating the flow field.

Through a hemolysis model, a relationship between these hydraulic results and the corresponding hemolytic response could be calculated. Several models for shear-induced hemolysis have been introduced and validated in the laminar regime, [6], however, a reliable and validated general hemolysis model for turbulent flow has yet to be developed.

\section{HEMOLYSIS CALCULATION AND ESTIMATION}

A popular hemolysis model is based on an empirical power law regression of the following form:

$$
D=C \tau^{\alpha} t^{\beta}
$$

where $D$ is some measure of damage, tipically the relative increase in plasma free hemoglobin $\triangle P f H b / H b, \tau$ is a constant shear stress (Pa) to which the blood sample is exposed for the duration $t$ (seconds). $C, a$ and $b$ are experimentally determined coefficients which have been presented by several investigators over the past 20 years. One popular regression is offered by Giersiepen et al. [6] in which $C=3.62 \times 10^{-7}$, $\alpha=2.416$, and $\beta=0.785$.

$$
\frac{\Delta P f H b}{H b}=\left(3.62 \times 10^{-7}\right) \tau^{2.416} t^{0.785}
$$

These regressions are commonly performed for experiments with uniform shear for a determined exposure time. Therefore, translation to practical problems in which shear may vary in space and time has required integration of Equation 1 along streamlines [7,8] or pathlines, which entails a Lagrangian tracking of a finite set of particles seeded in the flow path [5], or more recently, an Eulerian approach introduced by Garon and Farinas $[9,10]$, based on a transport equation derived directly from Equation 1 and ASTM hemolysis standards [2].

$$
\left(\frac{\partial}{\partial t}+\bar{U} \cdot \nabla\right) D_{L}=\left(3.62 \times 10^{-7}\right)^{1 / 0.785} \tau^{2.416 / 0.785}
$$

where $D_{L}$ stands for a local linear blood damage. This variable represents a measure of the hemolysis rate at each point of the computational domain. The development and simplifications of Equation 3 can be found with more detail at [9]. Garon and Farinas state that the accuracy of this Eulerian approach is related only with the precision of the CFD results, which depend basically on the mesh resolution and, in the case of turbulent flows, on the turbulence model used during the simulation.

The actual rheological behaviour of the blood is nonNewtonian, manifested in its shear-thinning and viscoelastic properties. Yeleswarapu et al. [11] have showed that these characteristics can be described quite well with a generalized Oldroyd-B fluid model. Previous authors performed CFD simulations of blood flow assuming a Newtonian-fluid behavior [3-5,8-10]. According to Mazumdar [1], this approximation is valid for shear rates higher than $50 \mathrm{~s}^{-1}$. For these shear rates, the blood has an asymptotic Newtonian behavior.

De Wachter et al. [12] used calf blood in their investigations. For comparative purposes, the same fluid was studied within this work. The asymptotic value of viscosity for the calf blood at high shear rates is $2.42 \mathrm{mPa} \mathrm{s}$ (cP), which is the same value used by De Wachter and Verdonck [7]

\section{TURBULENCE MODELLING}

Although the experimental setup of Wurzinger [6] only evaluates the laminar regime, other authors [13] have proved numerically and empirically the remarkable effect of turbulent stresses over hemolysis. This is an important subject of study, because in most of the biomedical devices, and particularly in the blood pumps, the flow regime is predominantly turbulent.

De Wachter et al. studied empirically [12] and numerically [7] the hemolysis in cannulas during a dialysis procedure. Figure 1 describes the geometry used in the numerical study. The inner diameters $A$ and $B$ stand for the cannula core and its tapered end, respectively. The Figure 1 also illustrates the different regions of the cannula nozzle studied by De Wachter and Verdonck. The dimensions of the different sizes of cannulas studied by them are listed in Table 1 . 


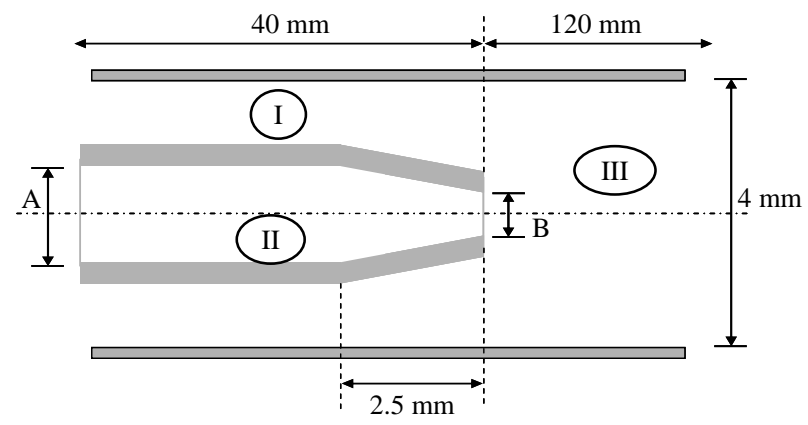

Figure 1. Dimensions of cannulas studied by De Wachter and Verdonck $[7,12]$, indicating regions within the annulus, within the cannula nozzle, and distal to the nozzle.

Table 1. Dimensions of the cannulas in Figure 1.

\begin{tabular}{ccc}
\hline & $\mathrm{A}(\mathrm{mm})$ & $\mathrm{B}(\mathrm{mm})$ \\
\hline $13 \mathrm{G}$ & 2.21 & 1.6 \\
$14 \mathrm{G}$ & 1.91 & 1.3 \\
$16 \mathrm{G}$ & 1.45 & 1.2 \\
\hline
\end{tabular}

De Wachter and Verdonck [7] specified an operational range of blood flow through the cannulas in a dialysis procedure, with a maximum of about $400 \mathrm{ml} / \mathrm{min}$, and a physiological range of blood flow through the artery between 500 and $1200 \mathrm{ml} / \mathrm{min}$. Based on the maximum value of these flow ranges, the average Reynolds number can be calculated using the characteristic dimensions for each region. In the case of the Cannula and Vessel region, the characteristic dimensions are the diameters of the cannula and the vessel, respectively. For the Annular region, the characteristic length is the hydraulic diameter of the annulus. These dimensions and corresponding average-Reynolds numbers are reported in Table 2, and indicate that transition from laminar to turbulent regime may occur within the domain under study. This transition was not considered in the previous numerical work by De Wachter and Verdonck [7], where they solved the laminar steady-state Navier-Stokes equations.

On the other hand, Kameneva et al. [13] have studied numerically and experimentally the blood capillary described in Figure 2. They analyzed both laminar and turbulent regimes. Their results showed a prominent effect of turbulence onto hemolysis rate. Turbulent stress, if exist, normally overcomes several times the magnitude of shear stress. Therefore, an accurate modeling of the turbulence is quite relevant, due to the high amount of uncertainty added to the flow solutions by the selection of different turbulence models.

\section{TURBULENCE BENCHMARK}

Based on the geometrical characteristic of cannulas and the capillary, a benchmark to validate the choice of the turbulence model has been selected. Balarac and Métais [14] performed a Direct Numerical Simulation (DNS) for an array of coaxial jets, which resembles closely the characteristics of the flow at cannulas, as it can be seen in Figure 3. In both cases, there is a shear layer between the inner and outer streams. In the turbulence benchmark, there is an additional shear layer between the outer jet and the free stream. This shear layer is not present on the cannulas, but the vessel's wall produces a boundary layer which has an effect similar to the lag caused by the low-speed free stream over the benchmark's outer jet.

On the benchmark, a laminar velocity profile is prescribed at the inlet of a rectangular parallelepiped domain. The shape of that profile is according to Equation 4.

$$
U_{x}(r)=\left\{\begin{array}{l}
\frac{U_{1}+U_{2}}{2}+\frac{U_{2}-U_{1}}{2} \cdot \tanh \left(\frac{r-R_{1}}{2 \theta_{01}}\right) \text { if } \quad r<R_{M} \\
\frac{U_{2}+U_{3}}{2}-\frac{U_{2}-U_{3}}{2} \cdot \tanh \left(\frac{r-R_{2}}{2 \theta_{02}}\right) \text { if } \quad r>R_{M}
\end{array}\right.
$$

$U_{1}, U_{2}$ and $U_{3}$ stand for the average velocities at the inner jet, the outer jet and the free-stream, respectively. $R_{1}$ and $R_{2}$ are the radii of the inner and outer jet, respectively, as specified at Figure 4. $R_{\mathrm{M}}$ is the arithmetic average between $R_{1}$ and $R_{2} . \theta_{01}$ and $\theta_{02}$ are the inlet momentum thickness of the inner and outer shear layers, respectively.

Table 2. Average Reynolds number at maximum flow rate for the different cannula sizes.

\begin{tabular}{|c|c|c|c|c|c|c|c|}
\hline & \multicolumn{3}{|c|}{$\begin{array}{c}\text { Characteristic } \\
\text { Dimensions [mm] }\end{array}$} & \multicolumn{3}{|c|}{ Reynolds by Region } \\
\hline & & $13 \mathrm{G}$ & $14 \mathrm{G}$ & $16 \mathrm{G}$ & $13 \mathrm{G}$ & $14 \mathrm{G}$ & $16 \mathrm{G}$ \\
\hline \multirow{3}{*}{$\begin{array}{l}\widetilde{0} \\
\stackrel{0}{0.0} \\
\widetilde{\sim}\end{array}$} & I & 1.59 & 1.81 & 2.35 & 1158 & 1215 & 1314 \\
\hline & II & 2.21 & 1.91 & 1.45 & 1680 & 1943 & 2560 \\
\hline & III & 4 & 4 & 4 & 2783 & 2783 & 2783 \\
\hline
\end{tabular}

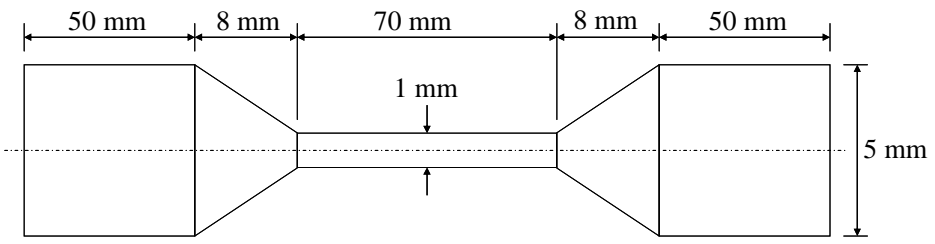

Figure 2. Dimensions of the capilar studied by Kameneva et al. [13].
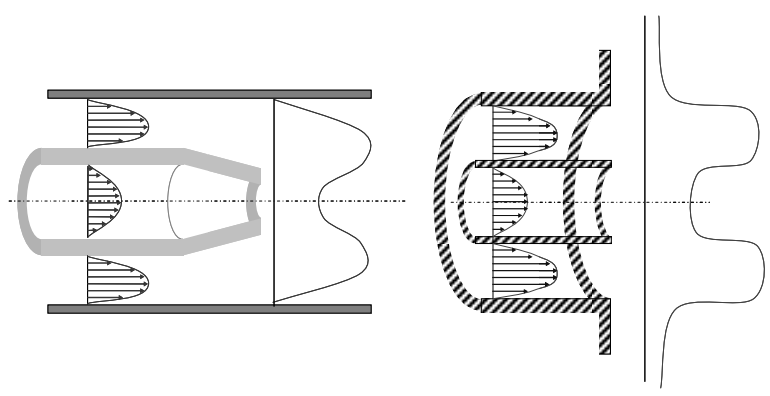

Figure 3. Similitude in the flow at cannula (left) and at the array of coaxial jets (right). 
Balarac and Métais used an array of jet with an outer-toinner diameter ratio of $R_{2} / R_{1}=2$, and a Reynolds number $\operatorname{Re}_{R_{1}}=2 U_{2} R_{1} / v=3000$. Also, the ratio of the jet outer radius to the outer shear layer momentum thickness at the inlet condition is fixed with $R_{2} / \theta_{02}=25$. The free-stream velocity was set to $U_{3} / U_{2}=0.04$. Further details about the boundary conditions used by Balarac and Métais can be found on [14].

Of all the cases and flow conditions studied by Balarac and Métais on their benchmark, the DNS 2_17 were selected. This case of study provides time-averaged information about the spreading rate and the axial velocity of the jet, which were reproduced here for comparison purposes. The parameters of this case are: $U_{2} / U_{1}=17$ and $R_{2} / \theta_{02}=12.5$. The plot of the inlet velocity profile (Equation 4 ) is showed at Figure 5.

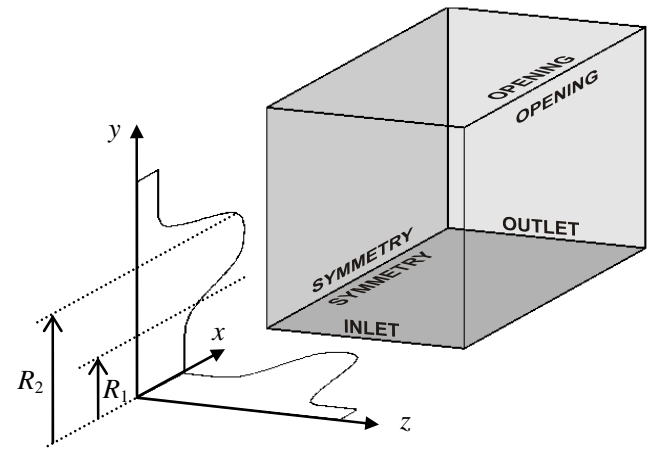

\section{Figure 4. Numerical domain used in the RANS solution of the coaxial jet array.}

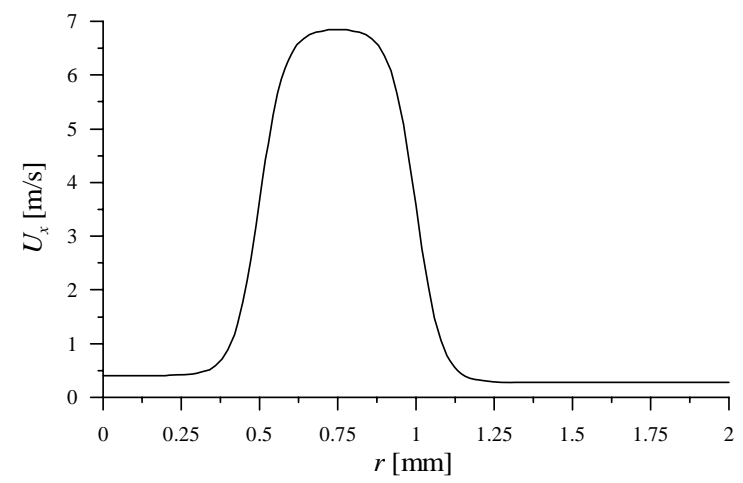

Figure 5. Laminar inlet velocity profile used during simulations.

Table 3. Grids size for the refinement study of the coaxial jets.

\begin{tabular}{cccc}
\hline Grid & $\begin{array}{c}\text { Transversal } \\
\text { Nodes }\end{array}$ & $\begin{array}{c}\text { Longitudinal } \\
\text { Nodes }\end{array}$ & $\begin{array}{c}\text { Total Control } \\
\text { Volumes }\end{array}$ \\
\hline n22_44 & 22 & 44 & 18963 \\
n28_55 & 28 & 55 & 39366 \\
n35_69 & 35 & 69 & 78608 \\
n44_87 & 44 & 87 & 159014 \\
\hline
\end{tabular}

\section{NUMERICAL PROCEDURE}

Hydrodynamic results of a jet array studied by Balarac and Métais were obtained with the commercial 3D finite volume code ANSYS CFX 10.0 (ANSYS Inc., Canonsburg, PA). This code uses finite volume for the discretization of the incompressible Reynolds-Averaged Navier-Stokes (RANS) equations [15]. For closure, the set of differential equations corresponding to the turbulence model is solved within the preceding numerical system.

There are many turbulence models that can be used in the simulation of turbulent flows. Among these, the most popular is the two-equation $k-\varepsilon$ model $[15,16]$, which use transport equations for the turbulence kinetic energy $k$ and turbulence eddy dissipation $\varepsilon$. The $k-\varepsilon$ model is known for being wellsuitable for predicting free-turbulent and high speed flows. Another popular turbulence model is the $k$ - $\omega$ model [15], which uses transport equations for $k$ and turbulence eddy frequency $\omega$. The $k$ - $\omega$ model is best suitable for turbulent boundary-layer and low-Reynolds number flows. Both models were tested within this work.

A most recent turbulence model with rising popularity is the Shear Stress Transport, or SST model [17]. This is basically a blending between the $k-\varepsilon$ and the $k-\omega$ models, according to the wall distance, behaving like $k-\omega$ near the wall, and like $k-\varepsilon$ on the free stream. The behavior of these models for transitional flows is not accurate, due to the complex phenomena of predicting the transition point. A transitional correction model, called Gamma-Theta, was developed by Menter et al. [18,19], based on two transport equations, one for the turbulence intermittency Gamma, and another for the transition onset in terms of the momentum thickness Reynolds number. These transport equations were solved within the SST turbulence equations system. The SST model and the SST with transitional Gamma-Theta model were also used within this work.

The flow was simulated for steady-state averaged conditions. Therefore, just one quarter of the benchmark geometry was simulated, considering a symmetry condition on the jet array, as shown on Figure 4.

By comparison between the DNS velocity profiles and the corresponding from the RANS simulation, the best suitable turbulence model was selected. The velocity at centerline of the jet, and the thickness of the jet were the comparison variables.

Figure 4 shows the structure of the numerical domain used for the solution of the RANS equations. The domain was discretized with a non-uniform hexahedral mesh. The hexahedral grid takes advantage of the polar symmetry of the jet array, working only with one-quarter of the domain, and with a higher density of elements and nodes in the regions near the $x$-axis. Table 3 presents the number of control volumes on each grid used in the refinement study. The first and second number in the grid nomenclature indicate the amount of nodes of that grid in the transversal and streamwise directions, respectively. 
The boundary conditions for the RANS simulations were set to resemble exactly those from the DNS simulations of [14]: a laminar inlet profile according to Equation 4 and as shown on Figure 5, with a ratio $R_{2} / R_{1}=2$ and a Reynolds number $\operatorname{Re}_{R_{1}}=3000$. The other parameters of Equation 4 were chosen to match the conditions from the DNS 2_17 case.

Although the inlet velocity profile is laminar, turbulence parameters should be specified, because a turbulence model is being used during the simulation. The most critical of these parameters is the turbulent-to-molecular viscosity ratio. The jet's spreading rate and development length are highly dependent on this parameter. A low viscosity ratio means a less turbulent flow at the inlet. With the $k-\varepsilon$ activated, this ratio was systematically lowered, reaching a value as low as possible, resulting in a numerically stable simulation $\left(v_{T} / v=0.05\right)$.

Higher viscosity ratios produces jets that develops turbulence too soon when are compared to DNS 2_17 benchmark. This value of the viscosity ratio was kept for the others turbulence models. The turbulence intensity was prescribed according to Equation 5.

$$
I T_{\text {in }}(r)=\left\{\begin{array}{ccc}
0.5 & \text { if } & 0.85 \cdot R_{1}>r \\
1.0 & \text { if } & 0.85 \cdot R_{1}<r<1.15 \cdot R_{1} \\
1.0 & \text { if } & 0.85 \cdot R_{2}<r<1.15 \cdot R_{2} \\
0 & & \text { everywhere else }
\end{array}\right.
$$

Using a standard $k-\varepsilon$ turbulence model, the streamwise $x$ component of the mean velocity vector $\left(U_{x}\right)$ at the centerline $(x-$ axis) was calculated for each grid. Also, the jet spreading rate was calculated. This is defined through a global shear layer thickness analogy, using the outer shear layer of the flow. Let $r_{\text {max }}(x)$ be the radial distance for which $U_{x}$ reaches its maximum value.

$$
U_{x_{\max }}(x)=\max \left[U_{x}(x, r)\right]=U_{x}\left(x, r_{\max }(x)\right)
$$

The thickness of the jet $\delta(x)$ is then defined as the radial distance such that

$$
U_{x}(x, \delta(x))=\frac{1}{2}\left[U_{x_{\max }}(x)-U_{3}\right]
$$

A sketch showing the physical representation of $\delta(x)$ is shown on Figure 6. For the different mesh sizes of Table 3, the values of $U_{x}$ and $\delta(x)$ as a function of the axial coordinate $x$ with the standard $k-\varepsilon$ turbulence model are plotted in Figure 7. As it can be seen, the curves are almost congruent, a signal of a sufficiently refined grid. Additional simulations were performed using the n44_87 grid with different turbulence models, specifically $k-\omega$, standard SST and SST with GammaTheta transition. The results are plotted on Figure 8, in comparison with the values of the DNS 2_17 case.

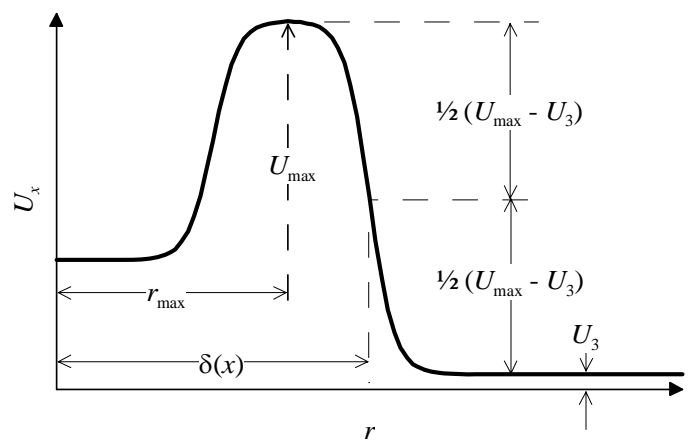

Figure 6. Physical representation of the jet spreading rate.
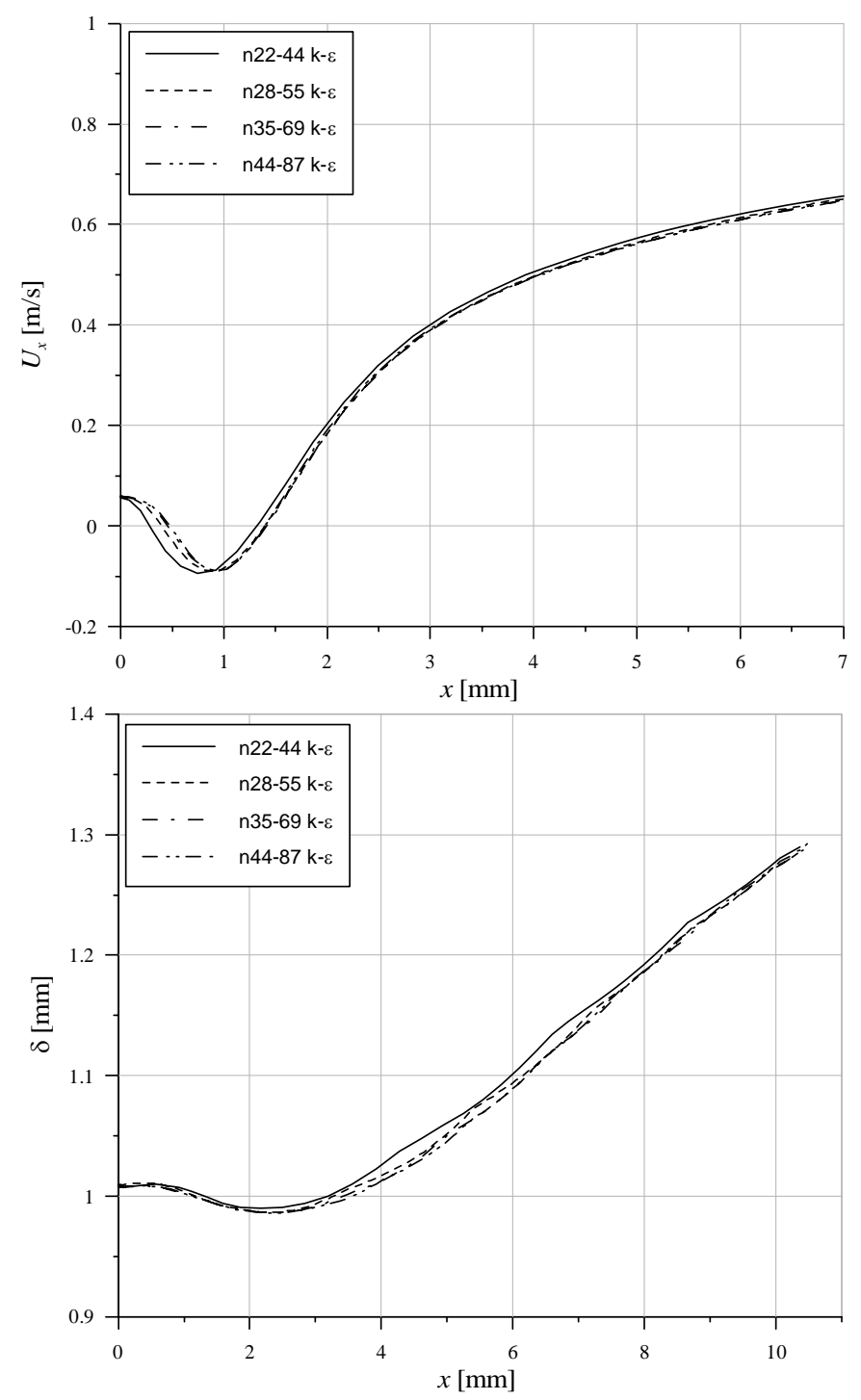

Figure 7. Results of refinement study for coaxial jets: centerline velocity (up) and jet radius (down) as a function of the axial coordinate $x$. 

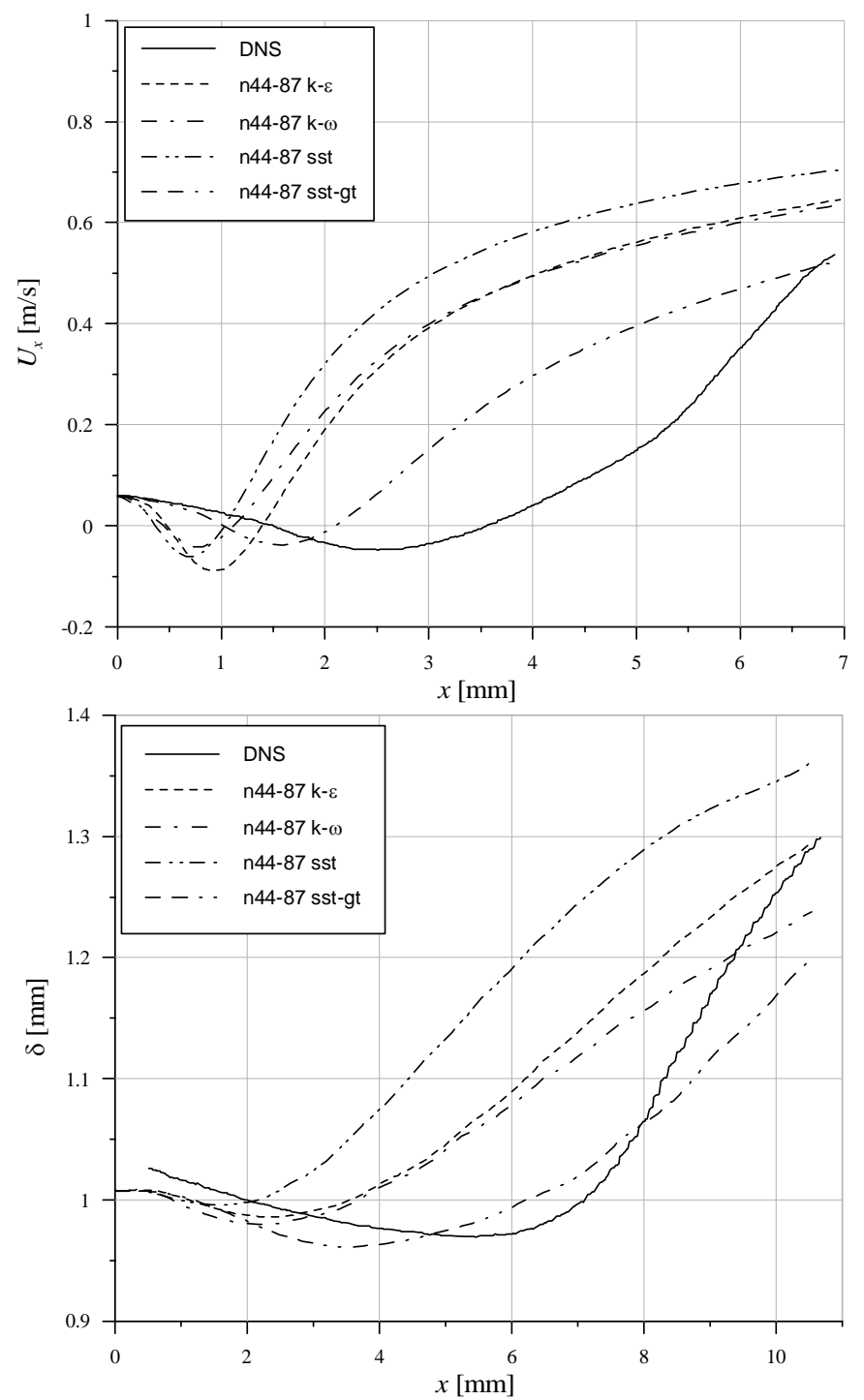

Figure 8. Results with different turbulence models: centerline velocity (up) and jet radius (down) as a function of the axial coordinate $x$.

\section{DISCUSSION AND CONCLUSIONS}

In this work, a simple validation procedure for a turbulence model to be used in cannulas and capillaries were developed. The accurate calculation of the turbulent stress becomes mandatory, especially when they have a predominant role at the numerical estimation of blood damage inside these biomedical devices

Different turbulence models $(k-\varepsilon, k-\omega$, SST and SST with Gamma-Theta transition) were found to significantly differ in predicting the flow development and the laminar to turbulence transition. Among the considered models, the one that better resembles the behavior of the jets DNS benchmark is the SST with Gamma-Theta transition, as it can be seen in Figure 8. The SST with Gamma-Theta transition model predicts quite well the evolution of the centerline velocity, with a correlation with the
DNS 2_17 curve closer than any of the other models studied. The spreading rate of the jet flow is also well described by the SST Gamma-Theta model. Therefore, this should be the choice for the simulation of cannulas, capillaries and other geometrically similar biomedical devices with mixed laminarturbulent conditions, and exist a development and transition of the flow from laminar to turbulent regime.

The use of a validated turbulence model diminishes the amount of uncertainty inherent to numerical blood-damage estimation. Further investigation into the hemolysis model and the comparison of the numerical result of different models with experimental values of hemolysis are currently under way.

\section{ACKNOWLEDGEMENT}

The authors want to acknowledge the financial support of this investigation by the Universidad Simón Bolívar's Deanship of Research and Development under a Research Assistantship grant.

Thanks also to the Laboratory of Fluid Mechanics at Universidad Simón Bolívar, whose PC cluster hosted a large number of the simulations performed in this investigation, and supplied the ANSYS CFX licenses for the study.

\section{REFERENCES}

1 Mazumdar, J.N., 1992, Biofluid Mechanics, World Scientific Publishing, Singapore.

2 ASTM. Designation: F 1841-97. Standard Practice for Assessment of Hemolysis in Continuous Flow Blood Pumps. Annual Book of ASTM Standards, Vol 13.01, 1997.

3 Burgreen, G.W., Antaki, J.F., Wu, Z.J., and Holmes, A.J., 2001, "Computational Fluid Dynamics as a development tool for rotary blood pumps”, Artif. Organs, 25(5), pp. 336-340.

4 Apel, J., Paul, R., Klaus, S., Siess, T., and Reul, H., 2001, "Assessment of Hemolysis Related Quantities in a Microaxial Blood Pump by Computational Fluid Dynamics”, Artif. Organs, 25(5), pp. 341-347.

5 Wu, J., Antaki, J.F., Snyder, T.A., Wagner, W.R., Borovetz, H.S. and Paden, B.E., 2005, "Design Optimization of Blood Shearing Instrument by Computational Fluid Dynamics”, Artif. Organs, 29(6), pp. 482-489.

6 Giersiepen, M., Wurzinger, L.J., Opitz, R. and Reul, H., 1990, "Estimation of shear stress-related blood damage in heart valve prostheses - in vitro comparison of 25 aortic valves”, Int. J. Artif. Organs, 13(5), pp. 300-306.

7 De Wachter, D., and Verdonck, P., 2002, "Numerical Calculation of Hemolysis Levels in Peripheral Hemodialysis Cannulas”, Artif. Organs, 26(7), pp. 576582.

8 Arora, D., Behr, M. and Pasquali, M., 2004, “A Tensorbased Measure for Estimating Blood Damage”, Artif. Organs, 28(11), pp. 1002-1015.

9 Garon, A. and Farinas, M.I., 2004, "Fast Threedimensional Numerical Hemolysis Approximation”, Artif. Organs, 28(11), pp. 1016-1025. 
10 Farinas, M.I., Garon, A., Lacasse, D. and N'dri, D., 2006, "Asymptotically Consistent Numerical Approximation of Hemolysis”, J. Biomech. Eng., 128(5), pp. 688-696.

11 Yeleswarapu, K.K, Kameneva, M.V., Rajagopal, K.R. and Antaki, J.F., 1998. "The flow of blood in tubes: theory and experiment”, Mechanics Res. Commun., 25(3), pp. 257262.

12 De Wachter, D.S., Verdonck, P.R., De Ros, J.Y. and Hombrouckx, R.O., 1997, "Blood Trauma in plastic haemodialysis cannulae”, Int. J. Artif. Organs, 20(7), pp. 366-370.

13 Kameneva, M.V., Burgreen, G.W., Kono, K., Repko, B., Antaki, J.F. and Umezu, M., 2004, "Effects of Turbulent Stresses upon Mechanical Hemolysis: Experimental and Computational Analysis”, ASAIO J., 50(5), pp. 418-423.

14 Balarac, G., and Métais, O., 2005, "The near field of coaxial jets: A numerical study”, Phys. Fluids, 17(6), pp. 065102/1-14.
15 ANSYS, Inc., 2005, ANSYS CFX-Solver, Release 10.0: Theory. ANSYS, Inc. Canonsburg, PA.

16 Launder, B.E., and Spalding, D.B., 1974, "The Numerical Computation of Turbulent Flows", Comp. Meth. Appl. Mech. Eng., 3, pp. 269-289.

17 Menter, F.R., 1994, "Two-equation Eddy-viscosity Turbulence Models for Engineering Applications”, AIAA J., 32(8), pp. 1598-1605.

18 Menter, F.R., Langtry, R.B., Likki, S.R., Suzen, Y.B., Huang, P.G., and Völker, S., 2004, "A Correlation Based Transition Model Using Local Variables: Part 1- Model Formulation” ASME-GT2004-53452, Proc. ASME Turbo Expo 2004, Vienna, Austria.

19 Menter, F.R., Langtry, R.B., Likki, S.R., Suzen, Y.B., Huang, P.G., and Völker, S., 2004, "A Correlation Based Transition Model Using Local Variables: Part 2- Test Cases and Industrial Applications” ASME-GT2004-53454, Proc. ASME Turbo Expo 2004, Vienna, Austria. 